\title{
ON AN IMPLEMENTATION OF THE
}

\section{MOHAN-ADIGA ALGORITHM}

\author{
Gisela Meister \\ GAO, Gesellschaft für Automation und Organisation mbH \\ Euckenstr.12, D - 8000 München, FR Germany
}

ABSTRACT. Several asymmetric cryptographic systems such as the RSA system [6] require modular exponentiation of large integers. This paper discusses a modular routine described in [2], which is suited for smart cards. It is based on the MohanAdiga algorithm [5]. This algorithm is comparatively fast, if the leading half of the bits of the modulus is -1. It will be shown that this restriction has some severe implications on the number of suitable primes and on the security of the system. If one decrements the number of leading I's then the security level of the system is increased while the speed is decreased.

\section{INTRODUCTION}

Software implementations of Public Key Algorithms based on modular exponentation are at present not very suited for say smart cards. This is due to the amount of time and RAM it takes to encipher a block or produce a digital signature for a document using a 512 bit modulus. In light of recent advances in factoring [4] it can be assumed that the length of the modulus and the execution time of the algorithm will be increased. This makes the need for a fast modular exponentiation method even more important. One approach to avoid the rather time-consuming bitwise reduction, often used in modular exponentiation [3], is the Mohan-Adiga algorithm which uses integer multiplication instead. Several authors ([1],[2],[5]) suggest that the speed be increased by choosing the modulus $m$ to satisfy

$$
2^{L M}-2^{L M / 2} \leq m<2^{L M}
$$


where LM denotes the length of the modulus in bits. This means that the leading half of the bits of $m$ is 1 . We assume LM to be a multiple of 4 .

One problem is of course to find suitable primes $p$ and $q$ which satisfy not only condition (1.1) but also those in [6]. In this paper it will be shown that, for a given prime $p$ of length $L M / 2$, there is at most one prime $q$ of length $L M / 2+1$ such that the product $m=p^{\star} q$ satisfies (1.1).

\section{CONSTRUCTION OF THE MODULUS}

Knobloch [2] uses the following approach to construct a modulus satisfying condition (1.1).

Let $p$ be an integer satisfying the following inequality

$$
2^{\mathrm{LM} / 2-1} \leq \mathrm{p}<2^{\mathrm{LM} / 2} \text {, }
$$

and let

$$
q_{0}=\left[\left(2^{L M}-2^{L M / 2}-1\right) / p\right]+1,
$$

where $[x]$ denotes the integer part of the real number $x$. Then

(2.3) $2^{\mathrm{LM} / 2+1}>q_{0} \quad 2^{\mathrm{LM} / 2} \quad$ and

(2.4) $2^{\mathrm{LM}}>\mathrm{p} * \mathrm{q}_{0} \geq 2^{\mathrm{LM}}-2^{\mathrm{LM} / 2}$.

This means that $m=p^{*} q_{0}$ satisfies condition (1.1).

In the prime number theorem [3, pp 366] it is proved that a given integer $p$ is prime with probability

$$
P(p)=1 / \ln 2^{\mathrm{LM} / 2} .
$$

In [2] it is stated that there exists a small intervall around $q_{0}$ which contains 'sufficiently many' primes $q$ such that the product $p^{*} q$ satisfies condition $(2.4)$. This is however not the case. For integers $q$ with $q<q_{0}$ or $q>q_{0}+1$ do not satisfy (2.4) as can easily be seen by substitution of $q_{0}$ by $q_{0}-1$ or $\mathrm{q}_{0}+2$, respectively.

Hence there is at most one candidate for $q$. This is $q_{0}$, if $q_{0}$ is odd, and $q_{0}+1$, if $q_{0}$ is even. If neither $q_{0}$ nor $q_{0}+1$ are prime, the process has to start again. 
In the sequel let $f(p)$ denote the odd candidate. It is straightforward to show by means of the prime number theorem that the conditional probability that $f(p)$ is prime, is bounded by

$$
P(f(p) \mid p)<1 / \ln 2^{L M / 2+1} .
$$

So the number of suitable pairs is

$$
\begin{aligned}
& P(p) * P(f(p) \mid p) * 2^{(L M / 2)} \\
& <A N Z=2^{L M / 2} /\left(\ln 2^{I M / 2} * \ln 2^{L M / 2}\right) .
\end{aligned}
$$

If $(p, q)$ does not have to satisfy $(2.4)$, we obtain

$$
P(p) \star P(q) * 2^{L M / 2} * 2^{L M / 2}=A N Z * 2^{L M / 2} .
$$

In $([1],[2],[5])$ it is stated that there are enough pairs of primes $(p, q)$ such that the product $m=p^{*} q$ satisfies (1.1). Note however that the number ANZ of pairs which satisfy (2.4) is equal to a quarter of the number of pairs with modulus of length $\mathrm{LM} / 2$ satisfying $2^{\mathrm{LM} / 4-1}<\mathrm{p}<2^{\mathrm{LM} / 4}$ and $2^{\mathrm{LM} / 4+1}>$ $\mathrm{q}_{0}>2^{\mathrm{LM} / 4}$.

The number of pairs is

$$
2^{\mathrm{LM} / 2} / \ln \left(2^{\mathrm{LM} / 4}\right)^{2}=\mathrm{ANZ} *(\mathrm{LM} / 2)^{2} /(\mathrm{LM} / 4)^{2}=4 \mathrm{ANZ} .
$$

\section{SECURITY RISKS}

Since there is a functional relation between $p$ and $f(p)$, we can obtain $p$ as the unique solution of the functional equation

$$
f(p) * p=m
$$

and the unique root of the function

$$
g(p)=m-p * f(p)
$$

For instance, equation (3.2) is easily solved if p satisfies $2^{\mathrm{LM} / 2}-2^{\mathrm{LM} / 4} \leq \mathrm{p}<2^{\mathrm{LM} / 2}$ or $2^{\mathrm{LM} / 4}-2^{\mathrm{LM} / 16} \leq \mathrm{p}<2^{\mathrm{LM} / 4}$. 
Say $p=2^{L M / 2}-x$ with $x<2^{L M / 4}$. Then $f(p)=2^{L M / 2}+x$ and

(3.2) reduces to the solution of the quadratic equation

$$
0=g(p)=m+2^{L M / 2+1} * p-p^{2}
$$

Therefore the approach suggested in [2] seems to be not feasible.

\section{MODIFICATION}

If we modify (1.1) to become

$$
2^{L M}>m \geq 2^{L M}-2^{L M * a}=L_{a}, 3 / 4 \leq a<1
$$

as suggested in [7], then the security of the system increases while the speed decreases. To be precise, we define
$(4.2)$

$$
q_{0}=\left[\left(L_{a} / p\right)\right]+1
$$

then the interval $I_{a}$, in which each $q$ together with $p$ satisfies (4.1), is
(4.3)

$$
I_{\mathrm{a}}=\left[q_{0}, q_{0}+2^{L M * a-L M / 2+1}\right]
$$

According to $[3, p 366]$ the number of its primes is roughly

$$
\text { (4.4) } \quad\left|I_{a}\right| / \ln 2^{L M / 2+1}
$$

and the probability that $q$ in $I_{a}$ is prime is equal to

$$
\text { (4.5) } \quad 1 / \ln 2^{\mathrm{LM} / 2}
$$

With increasing a the number ANZA of pairs, satisfying (4.1) increases by $2^{\operatorname{LM}}(a-1 / 2)$. It is

$$
A N Z A=A N Z *\left|I_{\mathbf{a}}\right|
$$

The closer a is to 1, the slower the Mohan-Adiga algorithm will be. So the optimal factor a depends on the security level as well as on the required speed of the algorithm. 


\section{BIBLIOGRAPHY}

[1] W.Fumy and A.Pfau, on the Complexity of Asymmetric Smart Card Authentication, Proceedings Smart Card 2000, 1989.

[2] H.-J.Knobloch, A Smart Card Implementation of the Fiat Shamir-Identificaton Scheme, in: Advances in CryptologyProceedings of Eurocrypt'88, Lecture Notes in Computer Science 330, Springer-Verlag 1988, 87-95.

[3] E.Knuth, The Art of Computerprogramming, vol.2, Seminumerical Algorithms, Addison-Wesley Publishing Company, Reading, 1980.

[4] A.K.Lenstra and A.K.Manasse, Factoring with two large primes, Abstracts of Eurocrypt 190.

[5] S.B.Mohan and B.S.Adiga, Fast Algorithms for Implementing RSA Public Key Cryptosystem, Electronic Letters (1985) vol. 21, no. 7, 761 .

[6] R.Rivest, A.Shamir and A.Adleman, A Method for obtaining Digital Signatures and Public Key Cryptosystems, Commun.ACM (1978) 120-126.

[7] C.Guillou and J.-J.Quisquater, Precautions Taken Against Various Potential Attacks in ISO/IEC DP 9796, Abstracts of Eurocrypt' 90 . 